\title{
Mechanical integrity of 18650 lithium-ion battery module: Packing density and packing mode
}

\author{
Binghe Liu ${ }^{\mathrm{a}, \mathrm{b}}$, Jinjie Zhang ${ }^{\mathrm{c}}$, Chao Zhang ${ }^{\mathrm{d}}$, Jun $\mathrm{Xu}^{\mathrm{a}, \mathrm{b}, \mathrm{e}, *}$ \\ a Department of Automotive Engineering, School of Transportation Science and Engineering, Beihang University, Beijing 100191, China \\ ${ }^{\mathrm{b}}$ Advanced Vehicle Research Center (AVRC), Beihang University, Beijing 100191, China \\ ${ }^{\mathrm{c}}$ School of Engineering, Beijing Forestry University, Beijing 100083, China \\ d Department of Structural Engineering, School of Aeronautics, Northwestern Polytechnical University, Xi'an, Shaanxi 710072, China \\ e State Key Laboratory of Nonlinear Mechanics (LNM), Institute of Mechanics, Chinese Academy of Sciences, Beijing 100190, China
}

\section{A R T I C L E I N F O}

\section{Keywords:}

Lithium-ion battery packs

Packing density

Packing mode

Finite element analysis

Mechanical integrity

\begin{abstract}
A B S T R A C T
The crash safety of lithium-ion batteries (LIBs) has recently become a hot research topic because of the wide application of LIBs in vehicle. This paper investigates how packing design of battery cells influence the energy density (volume specific) and structural of LIB pack. Firstly, three packing geometrical parameters (one packing angle parameter and two cell number parameters) are extracted to describe the packing modes, packing density and sizes of the module. Then a detailed computational model is established and validated through experiments, with the implementation of a failure criterion for short-circuit. An anisotropic elasto-plastic model is introduced to describe the mechanical response of the cylindrical jellyroll. Based on the computational results, we quantitatively describe the relationship between structural strength and packing parameters of battery module. The deduced empirical equations from the model are validated against numerical examples, and provide a reliable path to predict the mechanical integrity of battery packs with the knowledge of packing information. This developed modeling approach can serve as an efficient tool for safety design of LIB packs.
\end{abstract}

\section{Introduction}

Electric vehicles (EVs) are widely acknowledged as environment-friendly transportation devices because of their pollution-free nature and low petroleum consumption [1,2]. Among other candidate power sources, lithium-ion batteries (LIBs) have been widely used in EVs due to their relatively high energy and power density, high capacity, and long lifecycle [1,2]. Given the increasing number and proportion of available EVs on roads, the number of catastrophic accidents continues to increase due to the safety risks associated with LIB cells and packs [3-5], including electrical short-circuit, firing [6] and explosions [7,8].

Pioneering studies investigated the mechanical behaviors of LIB cells with various loading conditions [9,10] on pouch cells $[9,11,12]$. The mechanical behaviors of LIB components, such as cathodes [13], anodes [13], battery shells [9,14], and separators $[15,16]$, were also investigated comprehensively to understand the mechanical integrity of LIB cells as a structure. To satisfy engineering needs, Greve, and Fehrenbach [9] and Xu et al. [17] explored the quantitative relationship between the internal shortcircuit and mechanical stress status of LIBs caused by external mechanical loads which can describe the mechanical integrity of LIBs under various complicated loading conditions. Zhang et al. [18,19] studied the interaction between mechanical failure status and consequential electrical and thermal responses. These criteria have opened up a new area to examine the short-circuit phenomenon

\footnotetext{
* Corresponding author at: Department of Automotive Engineering, School of Transportation Science and Engineering, Beihang University, Beijing 100191, China. E-mail address: junxu@buaa.edu.cn (J. Xu).
} 


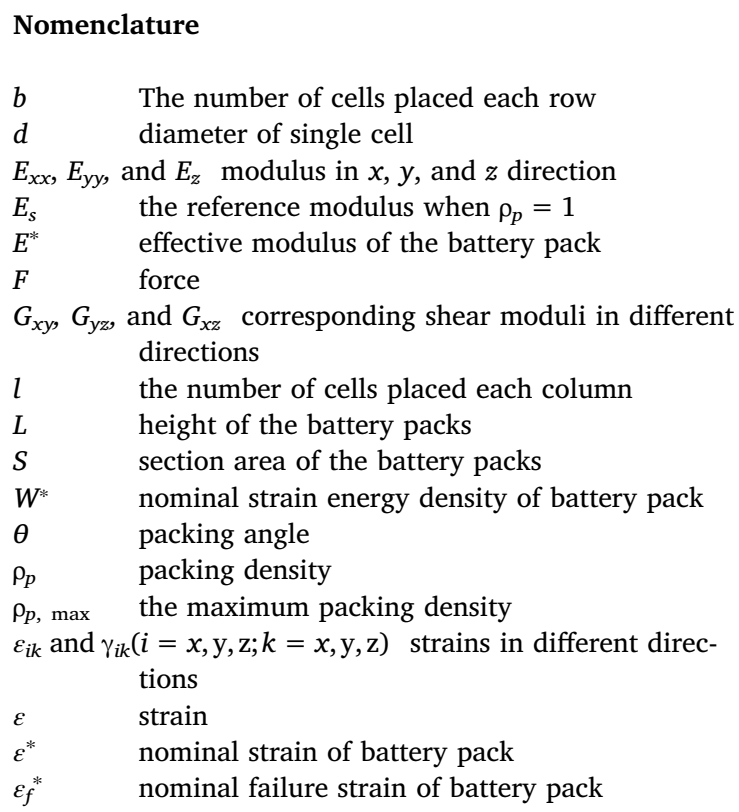

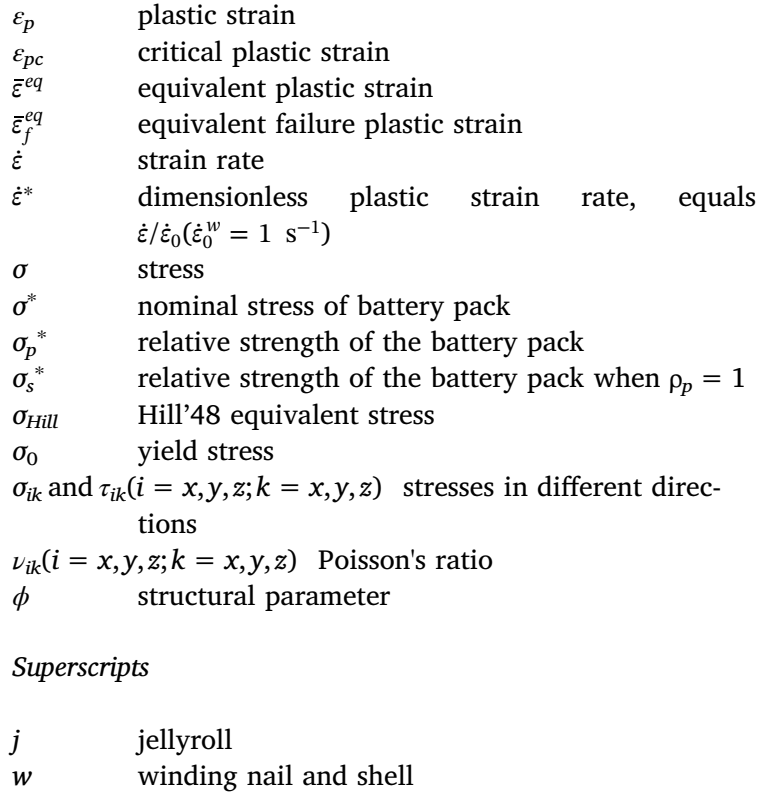

from a mechanical perspective. The vibration characteristics of LIBs were also experiment ally studied to understand the electrochemical performance of batteries in vehicles [20,21].

From the perspective short-circuit in a battery pack, Xia et al. [22] investigated the mechanical behavior of LIB square packs subjected to dynamic penetration from stone using a single-cell finite element model. Zhao et al. [23] studied the electrochemical behavior of nail penetrated LIB, and then developed a system to prevent thermal runaway for a square pack of LIBs. Some other studies employed various methods for detecting or monitoring short-circuit in situ to prevent thermal runaway [9,17] with the introduction of engineering-applicable devices to protect LIB packs from short-circuit [5]. Moreover, Nguyen et al. [24] and Kukreja et al. [25] preliminarily suggested new designs of battery module through enhancing the overall crash energy absorption capability of the module.

The safety of LIB packs highly depends on their mechanical integrity, which remains uninvestigated. The lack of research on this subject hinders the designing of better LIB packs. Given the light weight and small size of EVs, more space and weight limitations have been imposed on LIB packs such that the packing density and layout of these batteries have become prioritized and optimized targets. Trade-offs must be considered through battery safety, packing mode, and packing density. Therefore, the mechanical safety of LIB packs with various packing modes and densities must be investigated.

Based on the previous suggested mechanical model of a single battery [26], this paper establishes mechanical models for LIB packs with various packing modes and investigates the mechanical integrity of LIB packs upon previously correlated short-circuit criterion. This paper is organized as follows. Section 2 establishes a short-circuit criterion for single cell batteries and uses three parameters to characterize packing modes and pack sizes. Section 3 presents and analyzes typical numerical simulation results to demonstrate the mechanical integrity of LIB packs. Then how the mechanical integrity of these batteries changes across different governing parameter values is investigated and a method for rapidly predicting the mechanical responses of LIB packs is proposed. Section 4 concludes the paper.

\section{Methods}

In this work, we consider a rectangular battery module of cylindrical LIB cells (18650 cells). To study the pack design, we firstly develop a geometry model to describe the packing modes and packing sizes. Packing density, a measure of energy density of the battery module, is then calculated based on the geometry parameters. For mechanical integrity study, finite element method is used to predict the structural failure and initiation of short-circuit for LIB packs during a uniform constrained-compression condition.

\subsection{Geometrical model}

We examine cylindrical LIB cells (i.e., 18,650 LIBs), which may be regarded as the "circle packing in a square" problem from the perspective of geometry [27]. We focus on the " $b \times l$ " problem, where a number of $b$ and $l$ cells are placed in each row and column, respectively (to distinguish $b$ and $l$ more clearly, $l$ is defined as the number of cells in each column along the loading direction). Fig. 1 defines the packing angle $\theta \cdot \frac{\pi}{3} \leq \theta \leq \pi$ can be packed in several ways. Specifically, the cells in the same row do not come in contact with one another when $\frac{\pi}{3} \leq \theta \leq \frac{2 \pi}{3}$, but may touch one another when $\frac{2 \pi}{3}<\theta \leq \pi$ as shown in Fig. 1(a). 


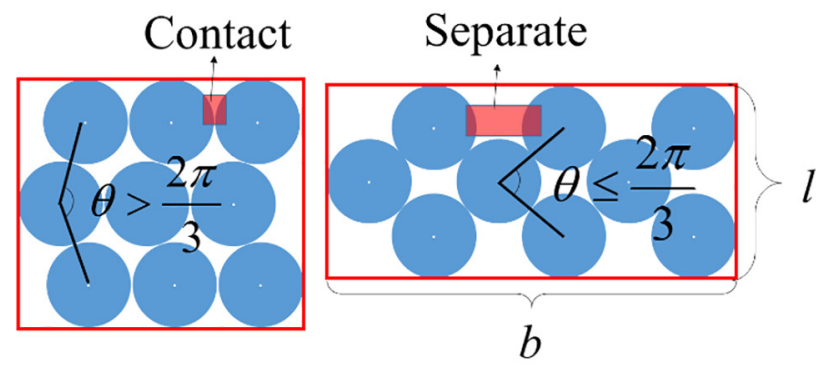

(a)

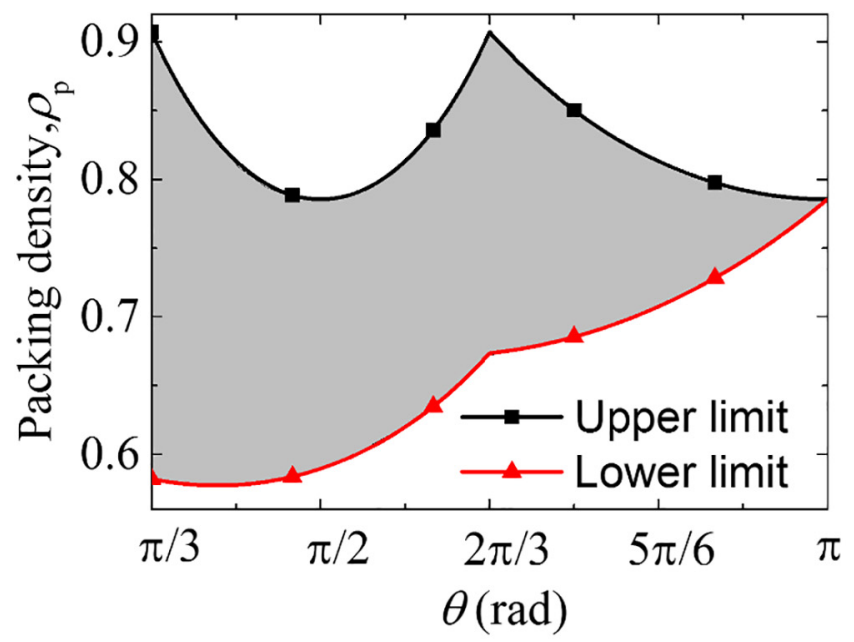

(b)

Fig. 1. (a) Packing modes and pack sizes defined by $b, l$, and $\theta$. (b) The upper and lower bounds of packing density against packing angle $\theta$ when $b=l=2$.

Packing density is defined as the volume density of battery cells in the whole module. It can be estimated through mathematical derivation as follows:

$$
\rho_{p}= \begin{cases}\frac{\pi b l}{4\left(2 b \cos \frac{\theta}{2}+1-\cos \frac{\theta}{2}\right)\left(l \sin \frac{\theta}{2}+1-\sin \frac{\theta}{2}\right)} & \left(\frac{\pi}{3} \leq \theta \leq \frac{2 \pi}{3}\right) \\ \frac{\pi b l}{4\left(b+\cos \frac{\theta}{2}\right)\left(l \sin \frac{\theta}{2}+1-\sin \frac{\theta}{2}\right)} & \left(\frac{2 \pi}{3}<\theta \leq \pi\right)\end{cases}
$$

When $b, \quad l \rightarrow \infty$, the theoretical maximum packing density can be obtained as:

$$
\rho_{p, \max }= \begin{cases}\frac{\pi}{4 \sin \theta} & \left(\frac{\pi}{3} \leq \theta \leq \frac{2 \pi}{3}\right) \\ \frac{\pi}{4 \sin \frac{\theta}{2}} & \left(\frac{2 \pi}{3}<\theta \leq \pi\right)\end{cases}
$$

As shown in Fig. 1(a), $\rho_{p}$, max varies from 0.785 to 0.907 , while packing density reaches its maximum value at $\theta=\pi / 3$ and $2 \pi / 3$ as well as its minimum value at $\theta=\pi$ and $\theta=\pi / 2$.

As from Eq. (1), packing density is directly related to $b$ and $l$, that is, $\rho_{p}$ increases along with the increase of either of them. The minimum value of $\rho_{p}$ varies from 0.5820 to 0.785 with $b=l=2$. The gray area in Fig. 1(b) represents the domain of packing density The range of packing density differs along with $\theta$. Specifically, packing density ranges from 0.582 to 0.907 when $\theta=\frac{\pi}{3}$, but becomes equal to 0.785 for any pack size when $\theta=\pi$. In sum, packing density is determined by $b, l$, and $\theta$.

\subsection{Finite element model for LIB packs}

\subsubsection{Objective LIB information}

This study uses commercialized 18,650 LIBs from SONY. Fig. 2(a) shows the geometrical picture and size of these batteries. These 


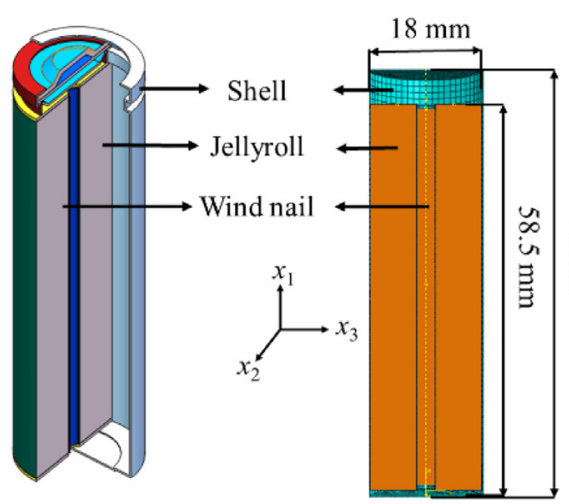

(a)
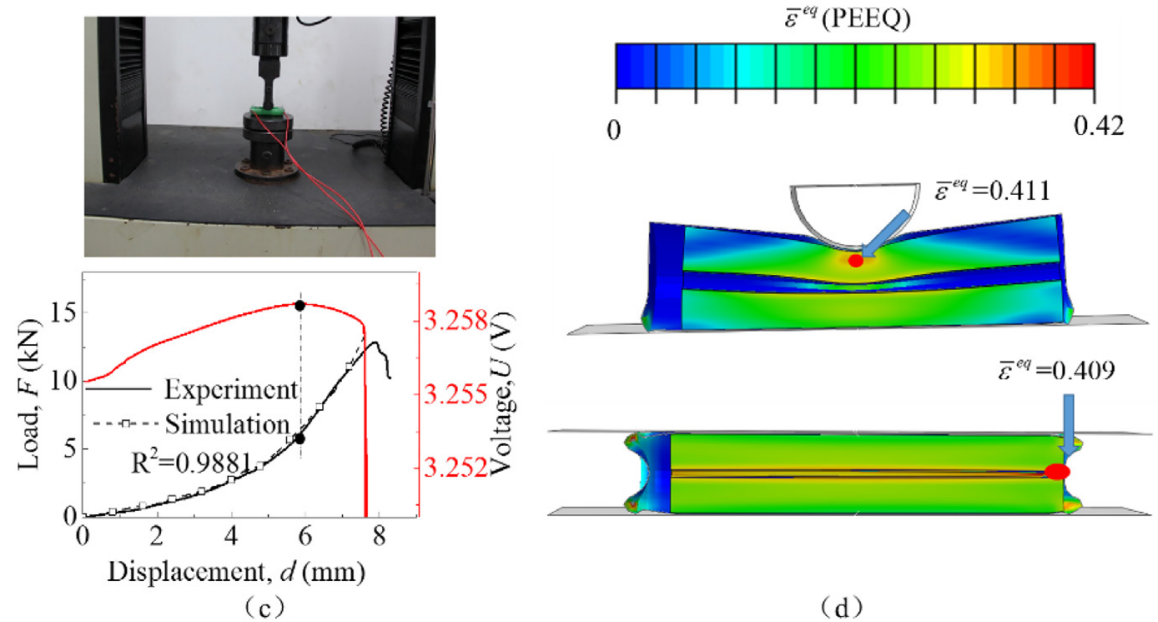

(d)

Fig. 2. (a) Geometric description of an LIB structure. (b) Computation and experiment results of single LIB in quasi-static loading condition. (c) Computation and experiment results of quasi-static indentation tests of single LIB. (d) Equivalent plastic strain status at the instant of short-circuit for compression and indentation.

LIBs contain jellyroll, shell, wind nail, and some short-circuit protection kits. The positive and negative electrodes of these LIBs are LiCoO2 and LixC6, while their maximum capacity reaches $2200 \mathrm{mAh}$.

\subsubsection{Mechanical model of a single LIB cell}

In our previous work, we developed mechanical models for jellyroll, shell, and winding nail, respectively [9,17,26]. An anisotropic model for jellyroll coupled with SOC (State of Charge) and dynamic effect was developed in Ref. [26] where the jellyroll was assumed as a macroscopic homogeneous material. The following engineering constants are used to describe the anisotropic elastic properties of the jellyroll [26]:

$$
\left\{\begin{array}{l}
\varepsilon_{x x} \\
\varepsilon_{y y} \\
\varepsilon_{z z} \\
\gamma_{x y} \\
\gamma_{y z} \\
\gamma_{z x}
\end{array}\right\}=\left[\begin{array}{cccccc}
\frac{1}{E_{x x}} & -\frac{\nu_{y x}}{E_{y y}} & -\frac{v_{z x}}{E_{z z}} & 0 & 0 & 0 \\
-\frac{\nu_{x y}}{E_{x x}} & \frac{1}{E_{y y}} & -\frac{v_{z y}}{E_{z z}} & 0 & 0 & 0 \\
-\frac{\nu_{x z}}{E_{x x}} & -\frac{\nu_{y z}}{E_{y y}} & \frac{1}{E_{z z}} & 0 & 0 & 0 \\
0 & 0 & 0 & \frac{1}{G_{x y}} & 0 & 0 \\
0 & 0 & 0 & 0 & \frac{1}{G_{y z}} & 0 \\
0 & 0 & 0 & 0 & 0 & \frac{1}{G_{z x}}
\end{array}\right\}\left\{\begin{array}{c}
\sigma_{x x} \\
\sigma_{y y} \\
\sigma_{z z} \\
\tau_{x y} \\
\tau_{y z} \\
\tau_{z x}
\end{array}\right\}
$$

We describe the anisotropic properties in the plastic stage using the following Hill's 48 yield stress criterion [26]: 


$$
\sigma_{H i l l}^{j}=\sqrt{\begin{array}{l}
F^{j}\left(\sigma_{22}^{j}-\sigma_{33}^{j}\right)^{2}+\mathrm{G}^{j}\left(\sigma_{33}^{j}-\sigma_{11}^{j}\right)^{2}+H^{j}\left(\sigma_{11}^{j}-\sigma_{22}^{j}\right)^{2} \\
+2 L^{j} \tau_{23}^{j}{ }^{2}+2 M^{j} \tau_{31}^{j}{ }^{2}+2 N^{j} \tau_{12}^{j}{ }^{2}
\end{array}}
$$

The hardening model for jellyroll is coupled with SOC and dynamic effect as follows:

$$
\sigma^{j}= \begin{cases}\left(B^{j} \varepsilon_{p}^{j^{j}}+\sigma_{0}^{j}\right) \mathrm{e}^{c i s O c} & , \varepsilon_{p}^{j} \leq \varepsilon_{p c}^{j} \\ {\left[\sigma_{c}^{j}+B^{j}\left(\varepsilon_{p}^{n^{j}}-\varepsilon_{p c}^{j}\right)^{j^{j}} \mathrm{e}^{j S O C}\right] \mathrm{e}^{D^{j \xi^{*}}}} & , \varepsilon_{p}^{j}>\varepsilon_{p c}^{j}\end{cases}
$$

Considering the fact that this paper focuses on the mechanical behavior of LIB packs before the onset of internal short-circuit and with the stable temperature during charging/discharging under mechanical loadings [9], the temperature factor does not included here.

An isotropic elastic-plastic model was proposed for winding nail and shell in Refs. [9, 17]. We choose the following Johnson-Cook model as the hardening model:

$$
\sigma^{w}=\left(\sigma_{0}^{w}+B^{w} \varepsilon_{p}^{w n^{w}}\right)\left(1+C^{w} \ln \dot{\varepsilon}^{* w}\right)
$$

where $\sigma_{0}{ }^{w}, B^{w}$, and $C^{w}$ are the parameters determined in Ref. [17]. Table 1 summarizes the parametric values for the mechanical models of 18650 cell.

Note that we only focus on the LIB with constant SOC in the quasi-static loading condition and ignore the dynamic and SOC effects. Also, this paper focuses on the mechanical behavior of LIB before short-circuit with little temperature changes. So the temperature dependent properties are not considered.

\subsubsection{Finite element model (FEM) of single LIB cell}

The commercial finite element software ABAQUS is used for the numerical study. The FEM of single LIB is constructed based on the actual geometry of the cell, while the tiny yet geometrically complex parts are simplified for meshing, computational convergence, and efficiency. The FEM of single cell contains jellyroll, winding nail, and shell, with geometry and element information summarized in Table 2. Note that the element size and element number are confirmed through divergence analysis for efficiency and accuracy.

\subsubsection{Mechanical integrity criterion of single LIB}

The mechanical integrity criterion is employed to monitor the internal short-circuit of LIBs subjected to an external mechanical loading. Previous studies used either stress- [9,17] or strain-based criteria [19] to predict the onset of short-circuit for LIBs.

In this work, only compression condition (in battery pack simulation) is considered and the local compression deformation is the main reason for internal short-circuit. The plastic strain (computed from Hill criterion) can be used to reflect the local deformation for LIB. Thus a plastic criterion is employed as the failure criterion of short-circuit:

$$
\bar{\varepsilon}^{e q}=\bar{\varepsilon}_{f}^{e q}
$$

Note that the computed plastic strain is related to the mesh sizes, where $\bar{\varepsilon}^{e q}$ will be larger in smaller mesh sizes (in some local places). This means $\bar{\varepsilon}_{f}^{e q}$ should be chosen larger in larger mesh sizes. In this study, the mesh size of the jellyroll is fixed to $0.4 \mathrm{~mm} \times 0.4 \mathrm{~mm} \times 0.4 \mathrm{~mm}$.

\subsubsection{Experiments for validating the failure criterion}

To validate the failure criterion, we first obtain the displacement at the onset of short-circuit through experiments. The strain/ stress status at an identified loading displacement is then numerically commutated and then is used to obtain the effective failure plastic strain.

We conducted both quasi-static compression and indentation experiments (the radius of the indenter is $10 \mathrm{~mm}$ ) for LIBs with

Table 1

Summary of material properties for 18650 cell.

\begin{tabular}{lll}
\hline Parameters & Jellyroll [26] & Winding nail and shell [17] \\
\hline Elastic constants & $E_{x x}{ }^{j}=1500 \mathrm{MPa}$ & $E^{w}=207 \mathrm{GPa}$ \\
& $E_{y y}{ }^{j}=E_{z z}=500 \mathrm{MPa}$ & \\
Poisson's ratio & $\nu_{x y}{ }^{j}=\nu_{x z}=0.15$ & $\nu^{w}=0.3$ \\
& $\nu_{y z}=0.3$ & \\
Yield surface & $(1,0.162,0.162,0.28,1.73,1.73)$ & $\sigma_{0}{ }^{w}=0.364 \mathrm{GPa}$ \\
Strain hardening & $\sigma_{0}^{j}=0.8 \mathrm{MPa}$ & $B^{w}=0.3706 \mathrm{GPa}$ \\
& $C^{j}=1.103$ & $n^{w}=0.3478$ \\
& $\varepsilon_{p c}{ }^{j}=0.2$ & $C^{w}=0.062$ \\
& $B^{j}=930 \mathrm{MPa}$ & \\
& $D^{j}=0.02$ & \\
& $n^{j}=3.4$ & \\
& &
\end{tabular}


Table 2

Mesh information of the FEM for one 18650 cylindrical cell.

\begin{tabular}{lllll}
\hline Component & Material & Thickness (mm) & Element type & Element size (mm) \\
\hline Jellyroll & & & C3D8R & $0.4 \times 0.4 \times 0.4$ \\
Winding nail & Steel & 0.2 & S4R & $0.38 \times 0.38$ \\
Shell & Steel & 0.3 & S4R & $1 \times 1$ \\
\hline
\end{tabular}

$\mathrm{SOC}=0$ to generalize of the failure criterion. The quasi-static experiments were performed using a INSTRON 8801 material testing platform as shown in Fig. 2(b). Force and voltage sensors were used in these experiments to monitor the mechanical and electrical responses. The temperature sensor is not used in this experiment, because the voltage signal can detect short-circuit accurately and there is little temperature changes before short-circuit. The loading rate was set to a constant of $2 \mathrm{~mm} / \mathrm{min}$, treated as quasi-static.

Fig. 2(b) and (c) show the force-displacement and voltage-displacement curves of compression and indentation tests where the black solid lines are the force curves and the red solid lines are the voltage curves. The dotted lines are the simulation results and they are both well fitted with experiments (the coefficient of determination $R^{2}>0.97$ ). Quasi-static indentation tests of single LIB computational results correlates better to the experiments than compression tests, because the tiny yet geometrically complex parts in both sides of the cell have been simplified for meshing. The short-circuit displacements in the compression and indentation tests

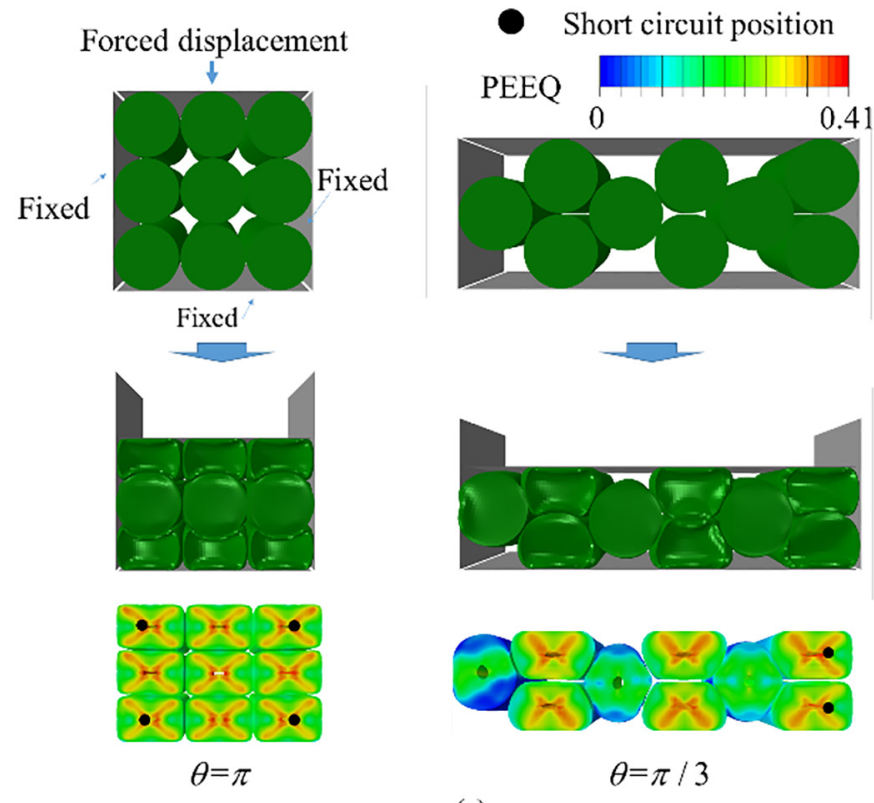

(a)

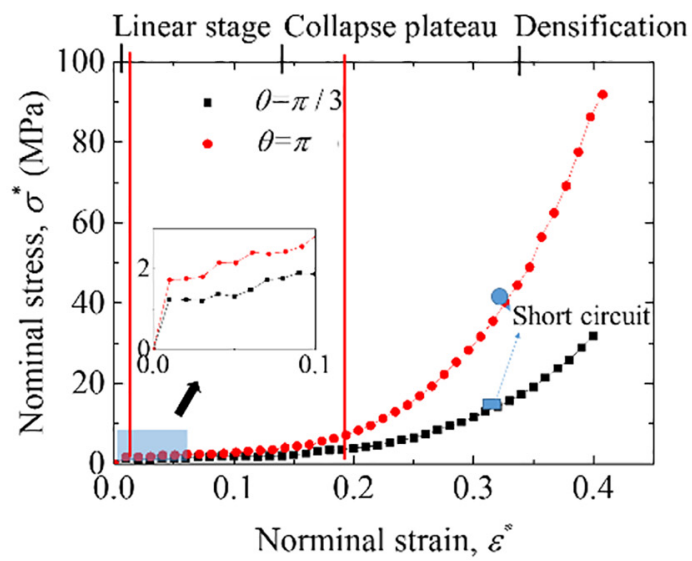

(b)

Fig. 3. (a) The loading condition and compression-deformed shape, and (b) the nominal stress-strain curve of a battery pack with $l=b=3$ and $\theta=\pi, \pi / 3$. 
$(\mathrm{SOC}=0)$ are identified experimentally to be $5.8 \mathrm{~mm}$ and $5.7 \mathrm{~mm}$, respectively. There is no big differences of the failure displacements between indentation and compression, because the radius of the indenter is not very small. The failure displacement will be smaller with smaller indenter. Calibrated by the numerical simulation of these two loading conditions, $\bar{\varepsilon}_{f}^{e q}$ is found to be 0.41 (jellyroll mesh size $4 \times 4 \times 4$ ) with SOC $=0$ in quasi-static conditions. As shown in Fig. 2(d), the occurrence of short-circuit (maximum equivalent plastic strain position) locates near the indenter in the indentation loading condition and locates at both ends of the cell under the compression loading condition. These experiment observations match well with those presented in previous references [9, 17].

\subsubsection{Loading condition for LIB packs}

For simplicity, a uniform constrained-compression loading condition for LIB packs is considered, which is the most frequently observed mechanical abuse condition for normal usage. In the FE analysis, the cells were packed together and two rigid plates were fixed to limit the lateral displacements of the LIB packs. Another rigid plate was fixed to limit the displacement in the vertical direction, while the fourth rigid body was given a constant displacement going down to compress the cells as shown in Fig. 3(a). A penalty-based contact was set for the components to avoid penetration with a friction coefficient of 0.1 . Note that the contact dynamics is not considered in this study, since the result is not sensitive with friction coefficient in this system. The explicit solver of ABAQUS was used with a relatively slow loading speed to represent the quasi-static situation.

\section{Results and discussions}

\subsection{Representative results}

The mechanical behavior of LIB packs can be predicted through numerical computation using the validated single cell model. Fig. 3 shows representative simulation results for a $3 \times 3$ battery pack of $\theta=\pi$ (corresponds to a packing density of 0.785 ) and $\theta=\pi / 3$ (corresponds to a packing density of 0.6631 ) in the quasi-static compression condition. The reaction force against displacement response shows a gradual stiffening process, similar to the force-displacement evolution profile of compression for a single cell $[13,26]$. The load-displacement curve is converted into the nominal stress-strain curve (Fig. 3 (b)), where $\sigma^{*}=F / S$ and $\varepsilon^{*}=d / L$ (with $S$ and $L$ denoting the section area and height of the battery packs, respectively). Similar to foam materials, the mechanical response of the two typical packing modes can be divided into a three-stage deformation process during compression [28]. As shown in Fig. 3(b), the red lines are the separations of the three stages. Stage I is the linear increasing stage; Stage II is the plateau stage with a gradually increasing stress, and Stage III is the densification stage where the battery pack deforms into a solid bulk "material". Short-circuit generally occurs in Stage III. However there are still some differences between these two packing modes e.g. structural stiffness, short-circuit strain, deformation and short-circuit positon. As shown in Fig. $3(\mathrm{~b}), 3 \times 3$ battery pack of $\theta=\pi$ has higher structural stiffness than $\theta=\pi / 3$ and the short-circuit strain. The short-circuit strain of $3 \times 3$ battery pack of $\theta=\pi$ and $\theta=\pi / 3$ are 0.33 and 0.32 separately. For $3 \times 3$ battery pack of $\theta=\pi$, cells at the four corners will be more prone to short-circuit. And for $3 \times 3$ battery pack of $\theta=\pi / 3$ cells more prone to short-circuit is at the right corners, shown in Fig. 3(a).

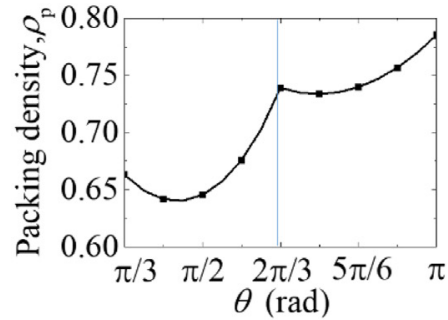

(a)

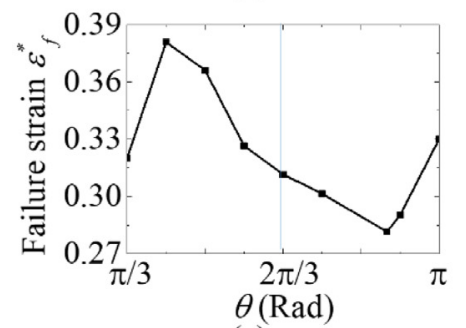

(c)

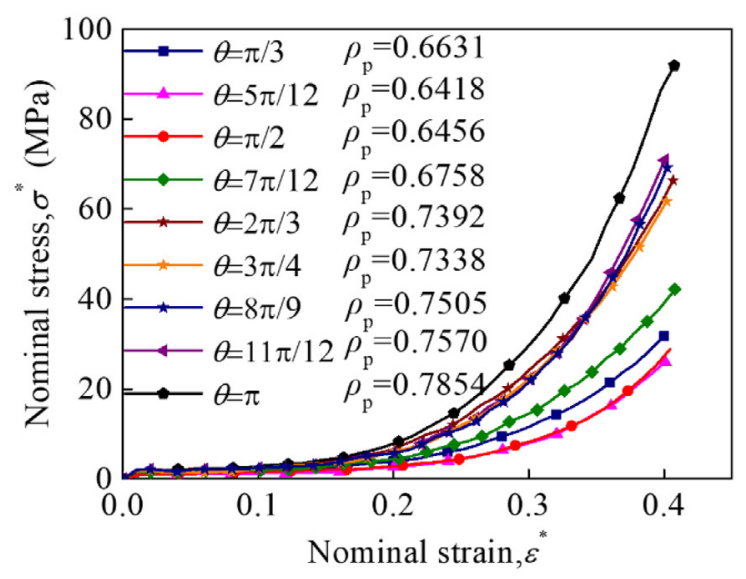

(b)

Fig. 4. (a) The relationship between packing density and $\theta$ when $b=l=3$. (b) The nominal stress-strain curve in various $\theta$ when $b=l=3$. (c) The relationship between failure strain and $\theta$ when $b=l=3$. 


\subsection{Packing mode effect}

Packing mode of a LIB pack is mainly determined by the parameter $\theta$. In this section, we investigate the mechanical integrity of battery packs with a $3 \times 3$ pack size $(b=l=3)$ and various packing angles $(\theta=\pi / 3,5 \pi / 12, \pi / 2,7 \pi / 12,2 \pi / 3,3 \pi / 4$, and $\pi)$. For the same pack size, packing density changes with the packing angle. Fig. 4(a) presents the packing density-packing mode curve. Overall, packing density increases with $\theta$, but slightly decrease for $\theta$ from $\pi / 3$ to $\pi / 2$ and from $2 \pi / 3$ to $3 \pi / 4$.

Fig. 4(b) shows the presence of a significant dependency of the mechanical response against packing mode. All packs show a similar nominal stress-strain response as described in previously for Fig. 4(b). Structural stiffness reaches its highest value when $\theta=\pi$, but reaches its smallest value when $\theta=5 \pi / 12 . \theta=\pi / 2$ and $\theta=5 \pi / 12$ have similar nominal stress-strain curves. Obviously, the packing mode with a higher packing density will be "stiffer" than that with lower packing density. However, those packing modes with similar packing densities may still demonstrate different mechanical behaviors (e.g., $\theta=3 \pi / 4$ and $\theta=2 \pi / 3$ ). Therefore, packing mode also determines mechanical behavior to some extent.

Fig. 4(c) shows the relationship between failure strain and packing mode for LIB packs of the same packing size. The failure strain increases as the packing angle increases from $\pi / 3$ to $5 \pi / 12$, decreases for $\theta$ from $5 \pi / 12$ to $8 \pi / 9$, and then increases again for $\theta$ from $8 \pi / 9$ to $\pi$. By comparing Fig. 4(c) and (a), we observe that the packing modes with a higher packing density have a smaller failure displacement when $\frac{\pi}{3} \leq \theta \leq \frac{2 \pi}{3}$, but reversely when $\frac{2 \pi}{3}<\theta \leq \pi$. This phenomenon may be attributed to the fact that $\theta=\frac{2 \pi}{3}$ is a critical packing mode, that is, the neighbor cells in the same row are not contact each another when $\theta>\frac{2 \pi}{3}$, but contact with each another when $\frac{\pi}{3} \leq \theta \leq \frac{2 \pi}{3}$ as shown in Fig. 4(a).

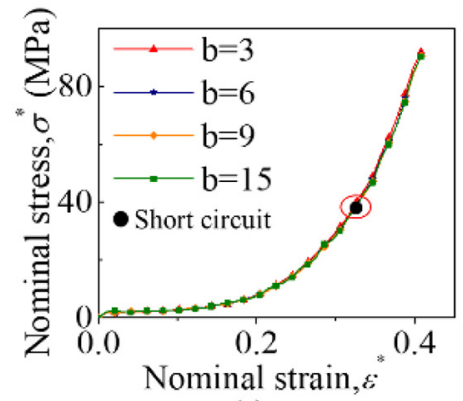

(a)

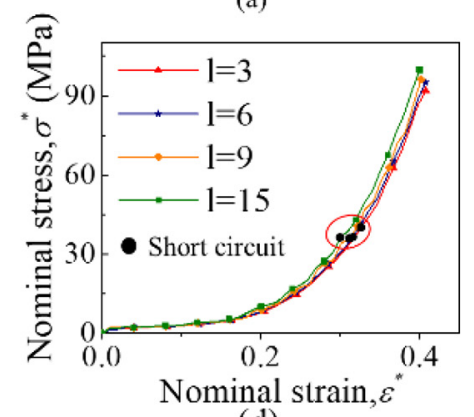

(d)

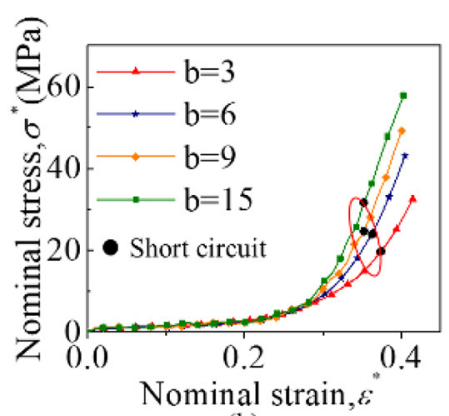

(b)

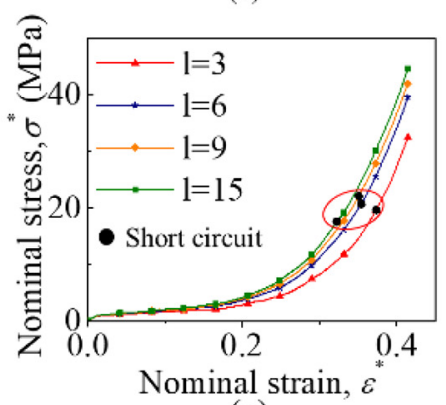

(e)

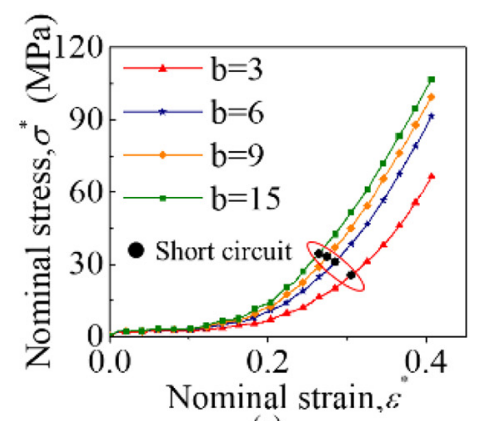

(c)

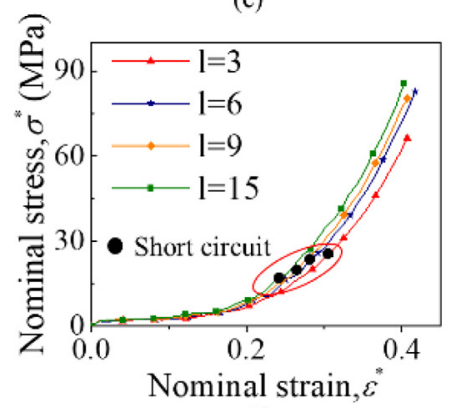

(f)

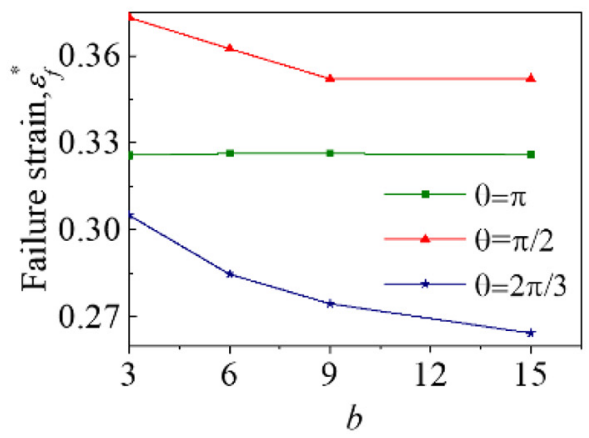

(g)

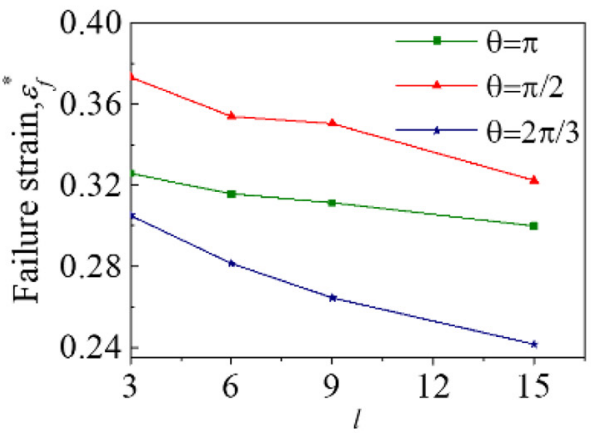

(h)

Fig. 5. The nominal stress-strain curves under compression for battery packs in (a) various $b, l=3$, and $\theta=\pi$, (b) various $b, l=3$, and $\theta=2 \pi / 3$, (c) various $b, l=3$, and $\theta=\pi / 2$, (d) $b=3$, various $l$, and $\theta=\pi$, (e) $b=3$, various $l$, and $\theta=2 \pi / 3$, and (f) $b=3$, various $l$, and $\theta=\pi / 2$, (g) and (h) show the relationship of failure strain with $b$ and $l$, respectively. 


\subsection{Size effect}

We use $b$ and $l$ to depict pack size and then choose three typical packing modes $(\theta=\pi, 2 \pi / 3$, and $\pi / 2)$.

When $\theta=\pi$, the packing density remains constant regardless of the values of $b$ and $l$, where $\rho_{p}=\frac{\pi b l}{4\left(b+\cos \frac{\theta}{2}\right)\left(l \sin \frac{\theta}{2}+1-\sin \frac{\theta}{2}\right)}=\pi / 4$. However, in some cases, the mechanical response of LIB packs slightly depends on their sizes. Although the mechanical behavior almost remains constant with $b$ (Fig. 5(a)) due to the uniform loading condition along the $b$ direction, Fig. 5(d) shows varying of nominal stress values for different $l$ that suggest the presence of size effect on the mechanical responses. Basically, a slightly larger nominal stress is observed for battery packs with a larger 1 . Similarly, the failure strain varies with $l$ but is almost the same for packs of different $b$ values as shown in Fig. $5(\mathrm{~g})$ and $(\mathrm{h})$.

When $\theta=2 \pi / 3$, both packing density and mechanical response change along with $b$ and l. In Fig. 5(b), the value of $l$ is fixed to 3 to investigate the influence of $b$, which varies by $3,6,9$, and 15 corresponding to pack density of $0.7392,0.7960,0.8170$, and 0.8346 , respectively. Unlike when $\theta=\pi$, a larger stress-strain curve is observed with a larger $b$ because of the changing packing density. The failure strain also shows a dependency on $b$ as shown in Fig. $5(\mathrm{~g})$. A larger $b$ indicates a smaller failure strain. Secondly, we set the value of $b$ to 3 to investigate the influence of $l$, which we set to $3,6,9$, and 15 with corresponding density values of $0.7392,0.7577$, 0.7641 , and 0.7693 , respectively (shown in Fig. 5(e)). The mechanical behavior for cases of $\theta=2 \pi / 3$ is strongly related to $l$ and shows a more significant dependency than that for the cases of $\theta=\pi$ because such behavior is affected by boundary condition (the different contact condition with the rigid plate) and packing density. The failure strain decreases with the increase of $l$ as shown in Fig. 5(h).

Similar to the cases of $\theta=2 \pi / 3$, the mechanical behavior for battery packs of $\theta=\pi / 2$ also show obvious dependency on $b$ and 1 . As shown in Fig. 5(c), (f), (g), and (h), the stress-strain curves for the battery packs become stiffer and the failure strain becomes smaller with larger $b$ and $l$.

\subsection{Packing-density-related mechanical behavior}

The preceding sections show the strong relationship of packing density with the mechanical behavior and failure strain of battery packs. Those battery packs with a high density are "harder" and have a smaller failure displacement than those with a small density.

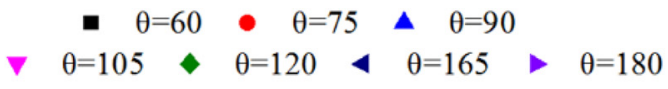

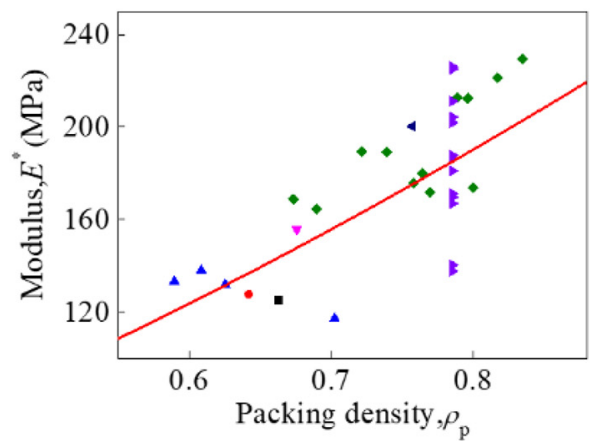

(a)

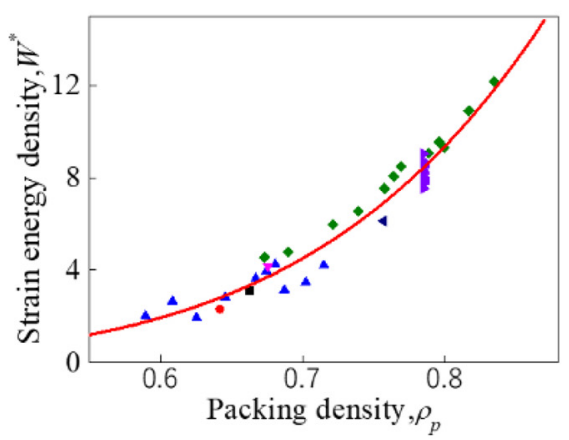

(c)

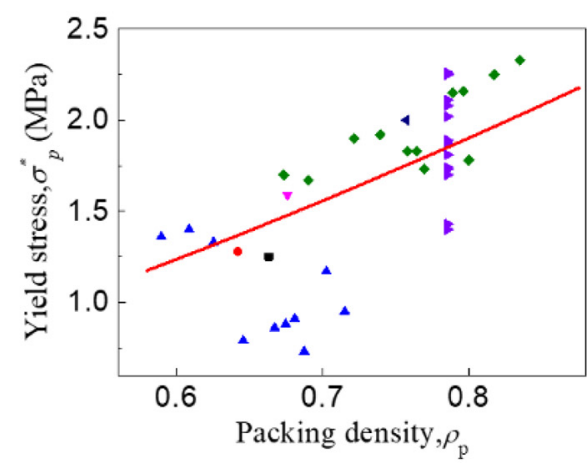

(b)

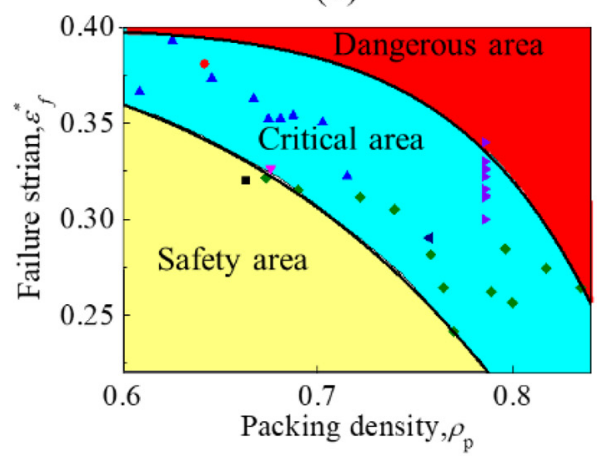

(d)

Fig. 6. The relationships between (a) modulus and packing density, (b) yield stress and packing density, (c) strain energy and packing densities and (d) failure strain and packing density. 
The battery packs under compression demonstrate the similar mechanical behavior of the aluminum-foamed material in Ref. [28], i.e. both responses follow a three-stage deformation process that includes liner range, plateau, and densification. The yield stress and elastic modulus vary across the different densities of the battery packs and the aluminum-foamed material. Thus, the battery packs may be treated as a "foam material" in terms of the mechanical behavior. Ashby [29] calculated the relationship among relative strength, elastic modulus, and density as follows:

$$
\frac{\sigma_{p}^{*}}{\sigma_{s}^{*}}=0.65\left(\phi \rho_{p}\right)^{3 / 2}+(1-\phi)\left(\rho_{p}\right)
$$

and

$$
\frac{E^{*}}{E_{s}^{*}}=C_{1}\left(\phi \rho_{p}\right)^{3 / 2}+C_{2}(1-\phi)\left(\rho_{p}\right)
$$

where $C_{1}$ and $C_{2}$ are the proportion constants that are fitted as 0.65 and 1 , respectively, and $\phi$ is the structural parameter that is equal to $1 . \sigma_{s}{ }^{*}$ and $E_{s}^{*}$ for the aluminum-foamed material can be obtained through testing. However, given that no packing mode may achieve $\rho_{p}=1$, both $\sigma_{s}{ }^{*}$ and $E_{s}{ }^{*}$ can only be fitted by the data, where $\sigma_{s}{ }^{*}=4 \mathrm{MPa}$ and $E_{s}{ }^{*}=400 \mathrm{MPa}$. Fig. 6(a) and (b) show the relationship between modulus and packing density and that between relative strength and packing density, respectively.

In the plateau and densification stages, the tangent stiffness of the battery packs increase with packing density. The strain energy density $W^{*}=\int \sigma^{*} \mathrm{~d} \varepsilon^{*}$ (where $\sigma^{*}$ and $\varepsilon^{*}$ denote stress and strain, respectively) is used to compare the hardening degrees of the LIB packs. Fig. 6(c) shows the relationship between strain energy and packing densities, which may be expressed as follows:

$$
W^{*}(\rho)=31.77 \rho_{p}^{5.477}
$$

Fig. 6(d) shows the relationship between failure displacement and packing density. The cells with a higher packing density presence a smaller failure density. The green area in Fig. 6(d) represents the critical zone for failure of battery packs. The upper and lower limit lines can be fitted as safety margin:

$$
\begin{aligned}
& \varepsilon_{f, \text { upper }}^{*}=-1.17\left(\rho_{p}\right)^{12}+0.4 \\
& \varepsilon_{f, \text { lower }}^{*}=-0.67\left(\rho_{p}\right)^{5.5}+0.4
\end{aligned}
$$

\subsection{Prediction of the mechanical response for LIB packs}

The preceding analyses (Sections 3.2-3.4) reveal the relationship of overall mechanical behavior with governing parameters $b, l, \theta$, and $\rho_{p}$. Furthermore, the Young's modulus and relative strength are quantitatively described using $\rho_{p}$ as follows:

$$
\begin{aligned}
\sigma^{*} & =2.6\left(\rho_{p}\right)^{3 / 2} \\
E^{*} & =260\left(\rho_{p}\right)^{3 / 2}
\end{aligned}
$$

The stress in the plateau and densification stages can be expressed as $\sigma^{*}=f\left(\varepsilon_{p}^{*}, b, l, \theta, \rho_{p}\right)$ and $\operatorname{simplified~as~} \sigma^{*}=g\left(\varepsilon_{p}{ }^{*}, \theta\right) k(l) m(b) n$ $\left(\rho_{p}\right)$, where $g\left(\varepsilon_{p}{ }^{*}, \theta\right)$ is the hardening curve without the boundary condition and packing density factors. $g\left(\varepsilon_{p}{ }^{*}, \theta\right)$ is computed as follows:

$$
g\left(\varepsilon_{p}^{*}, \theta\right)=A \varepsilon_{p}^{* l(\theta)}+\frac{\sigma_{\rho}}{k(l) m(b) n\left(\rho_{p}\right)}
$$

Therefore,

$$
\sigma^{*}=g\left(\varepsilon_{p}^{*}, \theta\right)=A k(l) m(b) n\left(\rho_{p}\right) \varepsilon_{p}^{* l(\theta)}+\sigma_{p}^{*}
$$

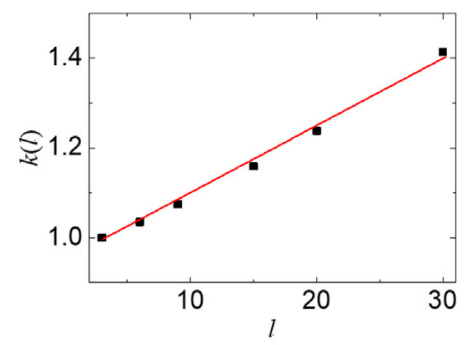

(a)

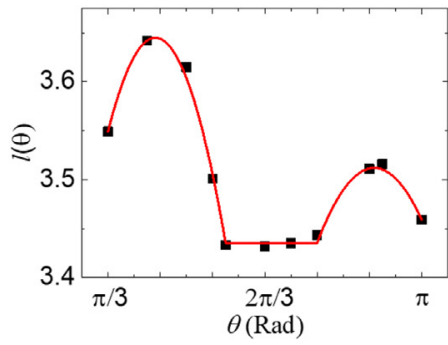

(b)

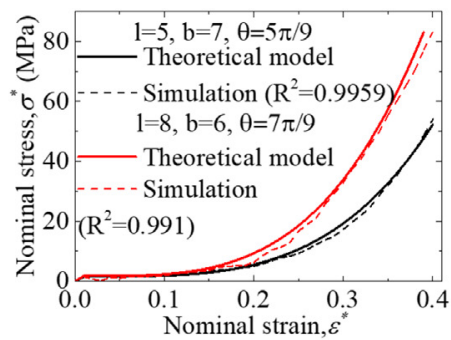

(c)

Fig. 7. (a) The fitting curve of $k(l)$. (b) The fitting curve of $l(\theta)$. (c) The compression simulation and theoretical model in random packs under compression. 
Dividing both sides of the above equation by $\sigma_{s}^{*}$, Eq. (16) can be written as:

$$
\frac{\sigma^{*}}{\sigma_{s}^{*}}=\frac{A}{\sigma_{s}^{*}} k(l) m(b) n\left(\rho_{p}\right) \varepsilon_{p}^{* l(\theta)}+0.65\left(\rho_{p}\right)^{3 / 2}
$$

where $k(l)$ and $m(b)$ are the boundary condition factors that can be calibrated by the packing mode of $\theta=\pi$ as follows:

$$
m(b)=1
$$

and

$$
k(l)=W^{*}(l \times 3, \theta=\pi) / W^{*}(3 \times 3, \theta=\pi)=0.0144 l+0.9568
$$

where $W^{*}(l \times 3, \theta=\pi)$ is the strain energy density when $b=3$ and $\theta=\pi$, and $W^{*}(3 \times 3, \theta=\pi)$ is the strain energy density when $l=3, b=3$, and $\theta=\pi$. Fig. 7(b) shows the fitting curve of $k(l)$.

$n\left(\rho_{p}\right)$ is the packing density factor that is determined based on the relationship of packing density against strain energy densities as shown in Fig. 7(d). It can be computed as follows:

$$
n\left(\rho_{p}\right)=\frac{W^{*}\left(\rho_{p}\right)}{W_{0}^{*}}=1.707 \rho_{p}^{5.477}
$$

where $W^{*}\left(\rho_{p}\right)$ is the strain energy density function with $\rho_{p}$, and $W_{0}^{*}$ is a reference strain energy density set as $W^{*}\left(\rho_{p}\right.$, $\max )=18.614 \mathrm{MPa}\left(\right.$ where $\left.\rho_{p, \max }=0.907\right)$.

$A / \sigma_{s}^{*}$ and $l(\theta)$ are fitted through the numerical results of various packing modes in $3 \times 3$ size, where $A / \sigma_{s}^{*}$ is calibrated as 1175.94. Fig. 7 (b) shows the relationship between $l(\theta)$ and $\theta$. From $\frac{\pi}{3}$ to $\frac{7 \pi}{12}$ and $\frac{7 \pi}{12}$ to $\pi, l(\theta)$ first increases and then decreases, and from $\frac{7 \pi}{12}$ to $\frac{7 \pi}{9}$. This varying trend can be expressed in the form of piecewise function as follows.

$$
l(\theta)= \begin{cases}3.645 \sin (0.7268 \theta+0.5798) & \frac{\pi}{3} \leq \theta \leq \frac{7 \pi}{12} \\ 3.4353 & \frac{7 \pi}{12}<\theta \leq \frac{7 \pi}{9} \\ 3.512 \sin (0.5487 \theta+0.0268) & \frac{7 \pi}{12}<\theta \leq \pi\end{cases}
$$

By integrating of Eqs. (17)-(21), one is capable to obtain explicit equation between the structural strength ( $\left.\sigma^{*}\right)$ and geometry parameters $(l, b$ and $\theta$ ), which can then be used to predict the mechanical integrity of a battery pack under constrained uniform compression loading condition. To validate the model, we randomly choose two packing forms with different sizes, modes, and densities, namely, $\theta=\frac{5 \pi}{9}$, where $l=5$ and $b=7$, and $\theta=\frac{7 \pi}{9}$, where $l=8$ and $b=6$. Fig. 7(c) shows a satisfactory agreement between the theoretical model and computation results $\left(\mathrm{R}^{2}>0.99\right)$, suggesting the accuracy and capability of the presented model in predicting the mechanical responses of battery packs. Therefore, the mechanical properties of one battery pack can be estimated simply through mechanical properties of single cell, packing model and packing size. This convenient method can be used to express the mechanical properties of battery pack in vehicle scale.

\section{Concluding remarks}

Finite element model is introduced to describe the mechanical behavior and short-circuit onset of 18,650 LIB packs, and the governing variables $l, b$, and $\theta$ are used to describe the packing modes and pack sizes. This paper examines the quantitative relationship among packing modes, pack sizes, and mechanical integrity of LIB packs.

Firstly, we examine the evolution of mechanical behavior across different $l, b$, and $\theta$. For battery packs of the same size, packing density is found to be the dominate parameter that a higher packing density corresponds to a higher structure stiffness. However, the failure strain for short-circuit has no obvious relationship with $\theta$ or packing density. For the same packing mode, the $b$ and $l$ combination of a larger packing density physically results in a stiffer but a smaller failure strain for the battery pack. In the same packing density, a change in $l$ does not affect the mechanical behavior, but an increase in $b$ makes the battery pack stiffer due to the boundary effect. The numerical results provide insights on the design and manufacture of LIB packs. Secondly, a foam-type material model is applied to predict the modulus and strength of battery packs, and is validated with numerical examples.

Results quantify the relationship of packing design and energy density of LIB packs, and gives a convenient method that can be used to express the mechanical properties of battery pack in full-vehicle scale, thus providing a promising path for the safety design of battery modules. In future works, the loading modes (e.g. axial compression/indentation/bending and uniaxial indentation/bending) and loading speeds effect (dynamic effect) will be discussed for better understanding the mechanical integrity of the battery packs.

\section{Acknowledgement}

This work is financially supported by The National Key Research and Development Program of China (2017YFB0103703), Research Project of the State Key Laboratory of Vehicle NVH and Safety Technology (NVHSKL-201610), Opening project of State Key Laboratory of Explosion Science and Technology (Beijing Institute of Technology) with project number of KFJJ17-13M and Scholarship from China Scholarship Council (CSC) with Grant number of 201706020109. 


\section{References}

[1] J.B. Goodenough, Y. Kim, Challenges for rechargeable Li batteries, Chem. Mat. 22 (3) (2010) 587-603.

[2] B. Dunn, H. Kamath, J.-M. Tarascon, Electrical energy storage for the grid: a battery of choices, Science 334 (6058) (2011) $928-935$.

[3] D. Miranda, C.M. Costa, A.M. Almeida, S. Lanceros-Mendez, Computer simulations of the influence of geometry in the performance of conventional and unconventional lithium-ion batteries, Appl. Energy 165 (2016) 318-328.

[4] J. Ye, H. Chen, Q. Wang, P. Huang, J. Sun, S. Lo, Thermal behavior and failure mechanism of lithium ion cells during overcharge under adiabatic conditions, Appl. Energy 182 (2016) 464-474.

[5] R. Zhao, J. Liu, J. Gu, Simulation and experimental study on lithium ion battery short circuit, Appl. Energy 173 (2016) $29-39$.

[6] A. Poullikkas, Sustainable options for electric vehicle technologies, Renew. Sustain. Energy Rev. 41 (2015) $1277-1287$.

[7] G. Zhang, F. Huang, Ieee, Research of the Electric Vehicle Safety Standard, 2012 World Automation Congress (Wac), (2012).

[8] P. Ping, Q.S. Wang, P.F. Huang, J.H. Sun, C.H. Chen, Thermal behaviour analysis of lithium-ion battery at elevated temperature using deconvolution method, Appl. Energy 129 (2014) 261-273.

[9] L. Greve, C. Fehrenbach, Mechanical testing and macro-mechanical finite element simulation of the deformation, fracture, and short circuit initiation of cylindrical Lithium ion battery cells, J. Power Sources 214 (2012) 377-385.

[10] B. Liu, S. Yin, J. Xu, Integrated computation model of lithium-ion battery subject to nail penetration, Appl. Energy 183 (2016) $278-289$.

[11] M.Y. Ali, W.-J. Lai, J. Pan, Computational models for simulations of lithium-ion battery cells under constrained compression tests, J. Power Sources 242 (0) (2013) 325-340.

[12] I. Avdeev, M. Gilaki, Structural analysis and experimental characterization of cylindrical lithium-ion battery cells subject to lateral impact, J. Power Sources 271 (2014) 382-391.

[13] W.J. Lai, M.Y. Ali, J. Pan, Mechanical behavior of representative volume elements of lithium-ion battery modules under various loading conditions, J. Power Sources 248 (2014) 789-808.

[14] X. Zhang, T. Wierzbicki, Characterization of plasticity and fracture of shell casing of lithium-ion cylindrical battery, J. Power Sources 280 (2015) 47-56.

[15] J. Xu, L. Wang, J. Guan, S. Yin, Coupled effect of strain rate and solvent on dynamic mechanical behaviors of separators in lithium ion batteries, Mater. Des. 95 (2016) 319-328.

[16] S.T. Yan, X.R. Xiao, X.S. Huang, X.D. Li, Y. Qi, Unveiling the environment-dependent mechanical properties of porous polypropylene separatorsle, Polymer 55 (24) (2014) 6282-6292.

[17] J. Xu, B. Liu, L. Wang, S. Shang, Dynamic mechanical integrity of cylindrical lithium-ion battery cell upon crushing, Eng. Fail. Anal. 53 (2015) 97-110.

[18] C. Zhang, S. Santhanagopalan, M.A. Sprague, A.A. Pesaran, Coupled mechanical-electrical-thermal modeling for short-circuit prediction in a lithium-ion cell under mechanical abuse, J. Power Sources 290 (2015) 102-113.

[19] C. Zhang, S. Santhanagopalan, M.A. Sprague, A.A. Pesaran, A representative-sandwich model for simultaneously coupled mechanical-electrical-thermal simulation of a lithium-ion cell under quasi-static indentation tests, J. Power Sources 298 (2015) 309-321.

[20] J.M. Hooper, J. Marco, G.H. Chouchelamane, C. Lyness, J. Taylor, Vibration durability testing of nickel cobalt aluminum oxide (NCA) lithium-ion 18650 battery cells, Energies 9 (4) (2016).

[21] T. Bruen, J.M. Hooper, J. Marco, M. Gama, G.H. Chouchelamane, Analysis of a battery management system (BMS) control strategy for vibration aged nickel manganese cobalt oxide (NMC) lithium-ion 18650 battery cells, Energies 9 (4) (2016).

[22] Y. Xia, T. Wierzbicki, E. Sahraei, X.W. Zhang, Damage of cells and battery packs due to ground impact, J. Power Sources 267 (2014) $78-97$.

[23] R. Zhao, S. Zhang, J. Gu, J. Liu, S. Carkner, E. Lanoue, An experimental study of lithium ion battery thermal management using flexible hydrogel films, J. Power Sources 255 (2014) 29-36.

[24] T.N. Nguyen, T. Siegmund, W. Tsutsui, H. Liao, W. Chen, Bi-objective optimal design of a damage-tolerant multifunctional battery system, Mater. Des. 105 (2016) 51-65.

[25] J. Kukreja, T. Nguyen, T. Siegmund, W. Chen, W. Tsutsui, K. Balakrishnan, H. Liao, N. Parab, Crash analysis of a conceptual electric vehicle with a damage tolerant battery pack, Extreme Mech. Lett.

[26] J. Xu, B. Liu, X. Wang, D. Hu, Computational model of 18650 lithium-ion battery with coupled strain rate and SOC dependencies, Appl. Energy 172 (2016) $180-189$.

[27] H.T. Croft, K.J. Falconer, R.K. Guy, Unsolved Problems in Geometry: Unsolved Problems in Intuitive Mathematics, Springer Science \& Business Media, 2012.

[28] Y.P. Jeon, C.G. Kang, S.M. Lee, Effects of cell size on compression and bending strength of aluminum-foamed material by complex stirring in induction heating, J. Mater. Process. Technol. 209 (1) (2009) 435-444.

[29] M.F. Ashby, A. Evans, N.A. Fleck, L.J. Gibson, J.W. Hutchinson, H. Wadley, F. Delale, Metal foams: a design guide, Appl. Mech. Rev. 23 (6) (2002) 119. 